\title{
A epidemia de AIDS no Brasil, 1991-2000: descrição espaço-temporal
}

\author{
The AIDS epidemic in Brazil, 1991-2000: space-time description
}

\author{
Antonio Luiz Rodrigues-Júnior ${ }^{1}$ e Euclides Ayres de Castilho²
}

\begin{abstract}
RESUM0
A incidência de AIDS foi retratada por um estudo ecológico dos municípios brasileiros, considerando as notificações entre 1991 e 2000. Observou-se aumento da incidência em mulheres e em indivíduos com baixa escolaridade. 0 Estado de São Paulo, com 46\% das notificações registradas no País, apresentou perfil epidemiológico distinto, quando a capital foi comparada com as cidades do interior; o Estado de Pernambuco apresentou um perfil epidemiológico semelhante àquele do início da epidemia no Brasil; o Estado Santa Catarina mostrou o predomínio de casos usuários de drogas injetáveis. 0 aumento de notificações pela via de transmissão ignorada indica que 0 viés de classificação foi crescente no sistema de vigilância epidemiológica.
\end{abstract}

Palavras-chaves: AIDS. Epidemiologia descritiva. Estudo ecológico. Vigilância epidemiológica. Brasil.

\begin{abstract}
The incidence of AIDS was described by an ecological study of Brazilian municipalities, considering cases reported from 1991 to 2000. An increasing incidence was observed in women and among individuals with a low educational level. São Paulo State had different epidemiological profiles when the State Capital was compared to other cities, with an overall $46 \%$ of reported cases in the Country; Pernambuco State had almost the same profile since the beginning of the epidemic in Brazil; In Santa Catarina State the category of intravenous drug users had the greatest exposure. The increasing number of cases reported under the category of unknown transmission showed the increased bias in measurement by the surveillance system.
\end{abstract}

Key-words: AIDS. Descriptive epidemiology. Ecological study. Surveillance. Brazil.

Na última década de 80, a epidemia de AIDS no Brasil atingia , principalmente, as regiões metropolitanas de São Paulo e do Rio de Janeiro, e os casos caracterizavam-se, em sua maioria, por serem do sexo masculino, por terem alto nível socioeconômico e por pertencerem às categorias de transmissão homossexuais/bissexuais, além dos casos portadores de hemofilia ou em receptores de sangue. A partir de 1990, constatou-se uma transição do perfil epidemiológico resultando na heterossexualização, feminização, pauperização e interiorização da epidemia ${ }^{3} 579$ 15. Nessa mudança de perfil, que se acentua ao decorrer o tempo, verifica-se: a) que a razão de sexo, que foi de 25 homens para cada mulher, em 1991, passou para 2 homens para cada mulher, em $2000^{4}$; b) a crescente ocorrência de casos em indivíduos com baixo grau de escolaridade ${ }^{11}$; c) 0 aumento do número de casos entre usuários de drogas injetáveis ${ }^{3}$.
Estudos descritivos sobre a epidemia podem ter abordagem geográfica, temporal e populacional ${ }^{13}{ }^{15}$, para caracterizar a mobilidade, a tendência e a vulnerabilidade, na observação dos fenômenos de transição do perfil epidemiológico. Tem-se descrito a AIDSno Brasil como sendo uma pandemia multifacetada, que é composta por várias subepidemias, não possuindo um perfil epidemiológico único em todo o território brasileiro, mas um mosaico de subepidemias regionais que são motivadas pelas desigualdades socioeconômicas².

0 objetivo deste trabalho é descrever o perfil epidemiológico da epidemia de aids, numa abordagem espaço-temporal dos casos notificados ao Ministério da Saúde do Brasil, diagnosticados entre 1991 e 2000.

\footnotetext{
1. Departamento de Medicina Social da Faculdade de Medicina de Ribeirão Preto da Universidade de São Paulo, Ribeirão Preto, SP. 2. Departamento de Medicina Preventiva da Faculdade de Medicina da Universidade de São Paulo, São Paulo, SP.

Endereco para correspondência: Dr. Antonio Luiz Rodrigues Júnior. FMRP/USP. Av. Bandeirantes 3900, 14049-900 Ribeirão Preto, SP.

Tel: $5516 \quad 602-2433$

e-mail: alrj@ fmrp.usp.br

Recebido para publicação em 16/10/2003

Aceito em 19/05/2004
} 


\section{MATERIAL E MÉTODOS}

Trata-se de estudo ecológico-social ${ }^{6} 1314$, adotando os municípios brasileiros como unidades de observação, representando a divisão político-administrativa do País adotada pelo Instituto Brasileiro de Geografia e Estatística (IBGE) em 1996 na contagem da população. Foram considerados os casos de AIDS notificados ao Ministério da Saúde, por ano de diagnóstico, entre $1^{\circ}$ de janeiro de 1991 e 31 de dezembro de 2000, do banco de dados da Coordenação Nacional de DST e AIDS do Ministério da Saúde, em 2002. Optou-se por não considerar os casos posteriores ao ano de 2000 em virtude do atraso de notificação. Foram consideradas, hierarquicamente, as seguintes categorias de transmissão: usuários de drogas injetáveis que compartilham agulhas e/ou seringas (UDI); homossexual/bissexual ( homo/bi); heterossexual ( hetero); transfusão de sangue/hemofilico ( sangue); perinatal (vertical) e ignorada (ignorado), de acordo com 0 critério de classificação do Ministério da Saúde em 2002. Foram consideradas, também, estratificações por sexo e por escolaridade, segundo número de anos de estudo: analfabeto; 1-3 anos; 4-7 anos; 8-11 anos; 12 ou mais anos; e ignorada. Recorreu-se ao Índice de Desenvolvimento Humano (IDH) como variável proxis do nível socioeconômico das unidades ecológicas, disponibilizado pelo Instituto de Pesquisa Econômica Aplicada (IPEA), em 1998.

Uma análise particularizada foi feita para os Estados de São Paulo, Pernambuco e Santa Catarina, por razões previamente conhecidas: São Paulo por deter o maior número de casos; Pernambuco, pela proporção expressiva de casos da categoria de transmissão homossexual/bissexual; e Santa Catarina, pela elevada proporção da categoria de usuário de drogas injetáveis.

\section{RESULTAD0S}

No período de estudo, foram notificados ao Ministério da Saúde 197.924 casos de AIDS. Em termos de coeficiente de incidência acumulada (por 10000 habitantes), os maiores valores foram: 74,8 em São Paulo, 64,5 em Santa Catarina, 39,5 no Rio Grande do Sul, 18,8 no Paraná, 12,0 no Rio de Janeiro e 11,7 em Minas Gerais. Ocorreram, no total, 41067 casos da categoria UDI. Em Santa Catarina, São Paulo e Rio Grande do Sul, observaram-se as maiores proporções de casos dessa categoria: 35\%, 28\% e 25\%, respectivamente. A Figura 1 apresenta a distribuição proporcional de casos no período em estudo, em cada categoria de transmissão, por estado, mostrando os diferentes perfis epidemiológicos da AIDS no País. Houve Estados que mostraram proporções maiores da categoria homossexual/bissexual, o que era uma característica comum do perfil epidemiológico no começo da epidemia, na década de 80, como observado em Pernambuco (39\%), no Ceará (39\%), no Pará (36\%), no Maranhão (35\%) e na Paraíba (33\%).

Pela análise da Tabela 1, depreende-se a razão de sexo em torno de cinco homens para cada mulher na categoria UDI, nos estratos de médio e alto desenvolvimento socioeconômico, e que a incidência nesta categoria não se alterou com o passar do tempo. Destaca-se o pequeno aumento de casos UDI em municípios do estrato de baixo desenvolvimento socioeconômico. Por outro

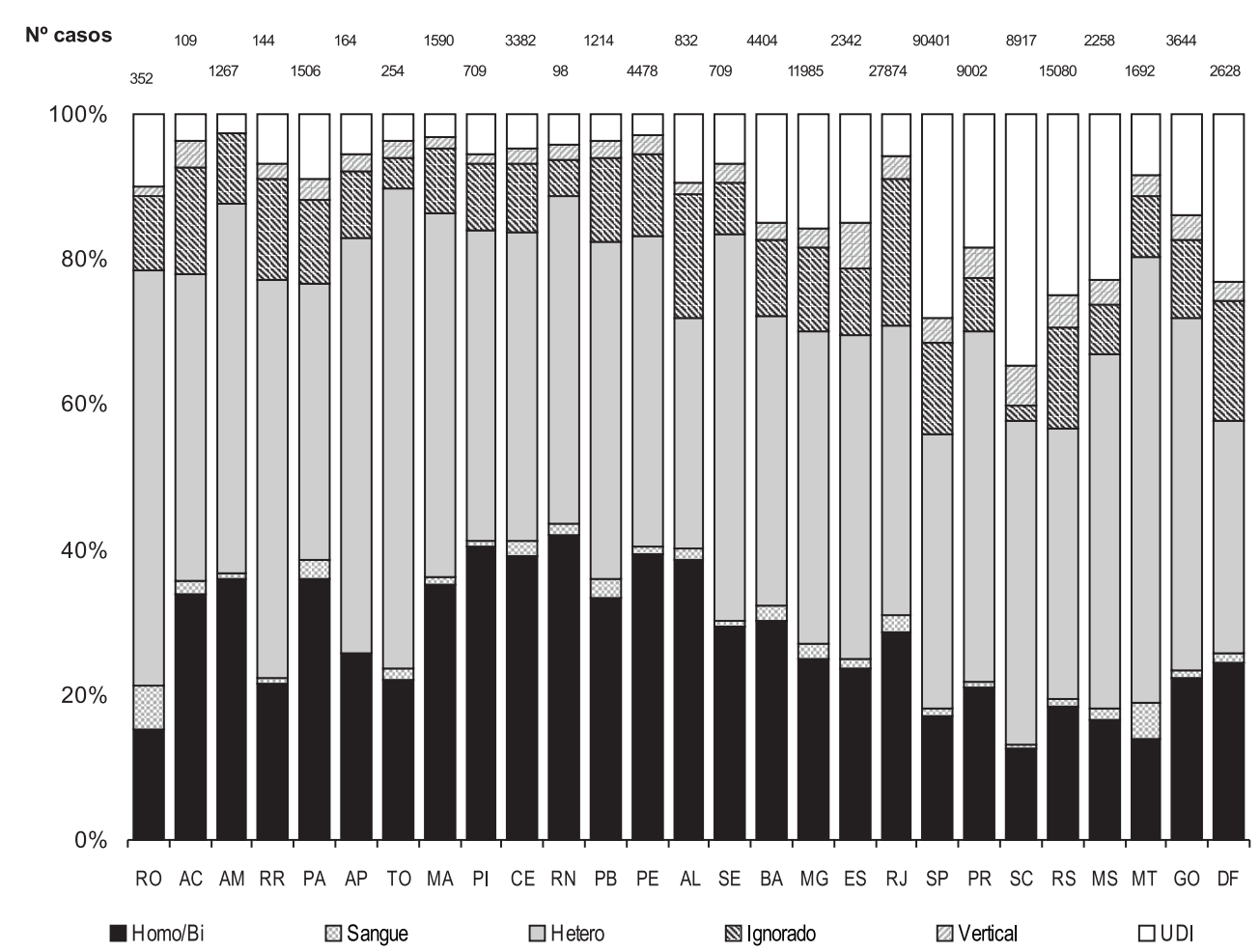

Figura 1 - Distribuiccão proporcional da incidência de aids por categoria de transmissão e por Unidade Federada. Brasil. 1991-2000. 
Tabela 1 - Distribuição da incidência de aids segundo via de transmissão, ano de diagnóstico, sexo e estrato de Índice de Desenvolvimento Humano dos municípios do Brasil. 1991-2000.

\begin{tabular}{|c|c|c|c|c|c|c|c|c|c|c|c|c|c|c|c|c|c|c|c|c|c|}
\hline \multirow[b]{2}{*}{ IDH } & \multirow[b]{2}{*}{ Categoria } & \multicolumn{2}{|c|}{1991} & \multicolumn{2}{|c|}{1992} & \multicolumn{2}{|c|}{1993} & \multicolumn{2}{|c|}{1994} & \multicolumn{2}{|c|}{1995} & \multicolumn{2}{|c|}{1996} & \multicolumn{2}{|c|}{1997} & \multicolumn{2}{|c|}{1998} & \multicolumn{2}{|c|}{1999} & \multicolumn{2}{|c|}{2000} \\
\hline & & masc & fem & masc & fem & masc & fem & masc & fem & masc & fem & masc & fem & masc & fem & masc & fem & masc & fem & masc & fel \\
\hline \multirow[t]{7}{*}{ Baixo } & UDI & 6 & - & 1 & - & 15 & 1 & 4 & 1 & 1 & - & 1 & 1 & 2 & 3 & 3 & - & 1 & - & 1 & \\
\hline & homo/bi & 38 & - & 29 & - & 24 & - & 38 & - & 14 & . & 22 & - & 12 & - & 15 & - & 13 & - & 4 & \\
\hline & sangue & 5 & 1 & 2 & - & - & 4 & 1 & - & 5 & 1 & 1 & 1 & 3 & 1 & - & - & - & - & - & \\
\hline & hetero & 11 & 14 & 13 & 7 & 21 & 17 & 22 & 13 & 24 & 27 & 25 & 23 & 23 & 19 & 19 & 30 & 21 & 20 & 7 & \\
\hline & vertical & 1 & - & 2 & - & - & 1 & & - & 1 & 1 & 2 & 3 & - & - & - & - & 2 & 1 & 1 & \\
\hline & ignorado & 9 & . & 15 & - & 11 & - & 13 & & 17 & - & 6 & - & 16 & - & 8 & - & 7 & & 5 & \\
\hline & btotal & 70 & 15 & 62 & 7 & 71 & 23 & 78 & 14 & 68 & 29 & 57 & 28 & 56 & 23 & 45 & 30 & 44 & 21 & 18 & \\
\hline \multirow[t]{7}{*}{ Médio } & UDI & 667 & 137 & 866 & 167 & 1037 & 228 & 989 & 190 & 1001 & 192 & 1078 & 248 & 1130 & 230 & 1056 & 210 & 927 & 182 & 772 & 13 \\
\hline & homo/bi & 939 & - & 1096 & - & 1188 & - & 1283 & - & 1267 & - & 1490 & - & 1567 & - & 1765 & - & 1542 & - & 1397 & \\
\hline & sangue & 89 & 19 & 78 & 34 & 76 & 28 & 65 & 37 & 78 & 44 & 82 & 55 & 73 & 28 & 19 & 4 & 12 & 3 & 12 & \\
\hline & hetero & 352 & 344 & 524 & 526 & 785 & 732 & 899 & 932 & 1155 & 1178 & 1476 & 1578 & 1835 & 2118 & 1933 & 2569 & 1959 & 2361 & 1848 & 239 \\
\hline & vertical & 23 & 30 & 35 & 34 & 60 & 39 & 53 & 41 & 45 & 81 & 62 & 94 & 111 & 105 & 129 & 122 & 103 & 116 & 76 & 7 \\
\hline & ignorado & 234 & 4 & 318 & 4 & 370 & 5 & 444 & 7 & 541 & 6 & 597 & 9 & 619 & 9 & 845 & 11 & 691 & 5 & 550 & \\
\hline & subtotal & 2304 & 534 & 2917 & 765 & 3516 & 1032 & 3733 & 1207 & 4087 & 1501 & 4785 & 1984 & 5335 & 2490 & 5747 & 2916 & 5234 & 2667 & 4655 & 261 \\
\hline \multirow[t]{7}{*}{ Alto } & UDI & 2432 & 422 & 2722 & 558 & 2775 & 537 & 2747 & 495 & 2837 & 485 & 2803 & 575 & 2678 & 529 & 2379 & 495 & 1811 & 376 & 1613 & 31 \\
\hline & homo/bi & 2744 & & 3197 & & 2905 & & 2965 & & 2928 & - & 2976 & & 3126 & - & 3238 & - & 2608 & & 2268 & \\
\hline & sangue & 202 & 70 & 176 & 78 & 190 & 81 & 165 & 70 & 186 & 90 & 196 & 63 & 108 & 53 & 28 & 6 & 19 & 2 & 25 & \\
\hline & hetero & 908 & 889 & 1343 & 1389 & 1637 & 1800 & 1891 & 2234 & 2178 & 2944 & 2438 & 3653 & 2795 & 4262 & 3002 & 4765 & 2662 & 4292 & 2665 & 4206 \\
\hline & vertical & 139 & 136 & 192 & 157 & 188 & 182 & 278 & 251 & 305 & 312 & 393 & 368 & 356 & 396 & 319 & 289 & 230 & 254 & 186 & 17 \\
\hline & ignorado & 891 & 12 & 1239 & 18 & 1564 & 11 & 1923 & 20 & 2541 & 21 & 2829 & 26 & 2195 & 15 & 2460 & 25 & 2095 & 24 & 1792 & 2 \\
\hline & subtotal & 7316 & 1529 & 8869 & 2200 & 9259 & 2611 & 9969 & 3070 & 10975 & 3852 & 11635 & 4685 & 11258 & 5255 & 11426 & 5580 & 9425 & 4948 & 8549 & 471 \\
\hline lal & & 9690 & 2078 & 11848 & 2972 & 12846 & 3666 & 13780 & 4291 & 15130 & 5382 & 16477 & 6697 & 16649 & 7768 & 17218 & 8526 & 14703 & 7636 & 13222 & 734 \\
\hline
\end{tabular}

lado, houve aumento da incidência em mulheres, na categoria de transmissão heterossexual. 0 número de casos de AIDS em mulheres superou o de homens em 1992, na categoria de transmissão heterossexual. No entanto, na categoria de transmissão ignorada, as notificações de casos do sexo masculino foram predominantes, mostrando a proporção de 14 mulheres para cada 1000 homens em 1991, 11 em 1994 e 15 em 2000. A estratificação por anos de estudo dos casos do sexo masculino, da categoria de transmissão ignorada, revela que, $85 \%$ dos casos apresentaram baixa escolaridade ( analfabeto, 1-3 e 4-7 anos de estudo) ou nível de escolaridade ignorada. 0 conteúdo da Tabela 2 mostra 0 aumento da proporção de casos nos estratos analfabeto, 1-3 e 4-7 anos de estudo, sendo respectivamente: $2 \%, 11 \%$ e $12 \%$ em $1991 ; 2 \%, 19,5 \%$ e $13 \%$ em $1994 ; 3 \%, 20 \%$ e 15\% em 1998; e, 3\%, 23\% e 15\% em 2000. Considerando 0 estrato de grau de instrução ignorada, observaram-se as seguintes proporções de notificações: 58\% em 1991, 47\% em 1994, 49\% em 1998 e 43\%, em 2000.

0 Estado de São Paulo teve 46\% das notificações. A Figura 2 dispõe as séries históricas dos números de casos de aids, segundo estratos de anos de estudos, comparando São Paulo com as demais Unidades Federadas. Observa-se, no Estado de São Paulo, o decréscimo de casos com escolarização ignorada, sendo 33\% em 1991, 27\% em 1995 e 22\% em 2000; identifica-se a inflexão na sequiência da incidência de casos com 1-3 anos de estudo em 1995, mostrando aumento, de 1991 (42\%) a 1994 (47\%), e decréscimo, de 1995 (43\%) a 2000 (31\%); houve aumento de casos com 4-7 anos de estudo, de 1994 (5\%) a 1998 (26\%). Para 0 conjunto das outras Unidades Federadas, 0 número de casos aumentou acentuadamente em todos os estratos de anos de estudo, exceto para 0 estrato com mais de 12 anos, que apresentou declínio de incidência entre 1991 (15\%) e 2000 (6\%); 0 aumento de casos de analfabetos é evidente, sendo de 3\% em 1991 e de 6\% em 2000; 0 estrato de 1-3 anos de estudo apresentou aumento discreto, de 1991 (3\%) a 1996 (8\%), e a rápida ascensão até $2000(32 \%)$.

№ Estado de São Paulo, a capital e as cidades do interior apresentaram características bem distintas. A capital apresentou 45.336 casos dos 90.401 do estado. Segundo a estratificação por escolaridade, os casos estão assim distribuídos na capital: $2 \%$ de analfabetos; $34 \%$ com $1-3$ anos de estudo; $13 \%$ com 4-7 anos; $14 \%$ com 8-11 anos; 8\% com mais de 12 anos; no interior as proporções foram $3 \%, 45 \%, 14 \%, 11 \%$ e $4 \%$, respectivamente. As proporções de casos com escolarização ignorada foram 30\% na capital e $22 \%$ no interior. Ocorreram, na categoria de transmissão ignorada, 8393 casos na capital e 3.089 no interior, sendo 8.320 e 3.067 casos do sexo masculino, respectivamente.

Tabela 2 - Distribuição da incidência deaids do sexo masculino da categoria de transmissão ignorada, estratificada por anos de estudo e ano de diagnóstico. Brasil. 1991-2000.

\begin{tabular}{lrrrrrrrrrrr}
$\begin{array}{l}\text { Anos de } \\
\text { estudo }\end{array}$ & 1991 & 1992 & 1993 & 1994 & 1995 & 1996 & 1997 & 1998 & 1999 & 2000 & Total \\
\hline Analfabeto & 17 & 31 & 63 & 58 & 92 & 86 & 102 & 95 & 88 & 81 & 713 \\
$1-3$ anos & 129 & 281 & 312 & 464 & 507 & 538 & 498 & 672 & 598 & 553 & 4552 \\
$4-7$ anos & 134 & 211 & 283 & 320 & 490 & 591 & 455 & 511 & 397 & 350 & 3742 \\
$8-11$ anos & 112 & 123 & 200 & 254 & 284 & 306 & 269 & 254 & 244 & 246 & 2292 \\
$\geq 12$ anos & 91 & 146 & 173 & 176 & 221 & 219 & 137 & 171 & 125 & 119 & 1578 \\
Ignorada & 667 & 802 & 929 & 1135 & 1531 & 1726 & 1392 & 1643 & 1369 & 1027 & 12221 \\
\hline Total & 1150 & 1594 & 1960 & 2407 & 3125 & 3466 & 2853 & 3346 & 2821 & 2376 & 25098
\end{tabular}


Dentre os casos do sexo masculino da categoria de transmissão ignorada, 3.901 na capital e 1.916 no interior, tinham registro ignorado sobre 0 grau de escolarização. A Figura 3 contempla a distribuição dos casos, no Estado de São Paulo, por categoria de transmissão. Na capital, houve decréscimo da incidência na categoria de transmissão homossexual/bissexual concomitante com a categoria de UDI; 0 número de casos na categoria de transmissão heterossexual aumentou até 1995, passando de 596 (19\%) em 1991, para 1.340 (33\%) em 1995; o número de casos na categoria de transmissão ignorada aumentou até 1995, sendo 297 (9\%) em 1991 e 851 (21\%) em 1995. No interior, o maior declínio foi verificado na categoria de UDI, que era de 2.091 (44\%) em 1993 e passou para 1.036 (20\%) em 2000; a incidência de casos na categoria de transmissão homossexual/ bissexual manteve-se, sendo 576 (18\%) em 1991, 745 (12\%) em 1996 e 641 (13\%) em 2000; 0 número de casos da categoria de transmissão ignorada foi menor que 0 da capital.

0 Estado de Pernambuco apresentou pequeno número de casos na categoria de UDI, como mostra a Figura 4: 3\% em 1991 e 2\% em 2000. As proporções de casos por transmissão homossexuais/bissexuais se destacaram: $54 \%$ em 1991 e 29\% em 1998. Verificou-se 0 aumento das proporções de indivíduos analfabetos e com 1-7 anos de estudo, que em 1991 foi de 21\% e em 2000 de 50\%. Houve pequeno acréscimo na parcela de casos da categoria de transmissão ignorada, sendo 9\% em 1991 e 13\% em 2000, dentre as quais 68\% em 1991 e
56\% em 2000 apresentaram grau de instrução ignorado, que podem ser do não preenchimento da ficha de notificação.

Em Santa Catarina, as proporções são diferentes daquelas de Pernambuco, como mostra a Figura 5. Houve decréscimo da proporção de casos na categoria de UDI, de 56\%, em 1991, para 22\%, em 2000; a incidência na categoria homossexual/bissexual diminuiu de 18\%, em 1991, para 13\%, em 2000; 0 número de casos na categoria de transmissão heterossexual aumentou de 21\%, em 1991, para 62\%, em 2000; e observou-se pequeno aumento na categoria de transmissão ignorada, de 0,6\% em 1991 para 1,6\% em 2000. Dos casos referentes à categoria de transmissão ignorada, não houve uma tendência definida, de aumento ou de decréscimo, em determinado estrato de anos de estudo.

\section{DISCUSSÃ0}

A tendência de maior número de mulheres na categoria de transmissão heterossexual, desde 1992, deve traduzir a maior vulnerabilidade feminina em relação à menor capacidade de negociar sexo seguro e à menor possibilidade de acesso aos serviços de saúde reprodutiva ${ }^{101112}$.

Acrescente proporção de casos da categoria de transmissão ignorada preocupa, pois, considerando que 0 sistema de vigilância epidemiológica está em processo de aprimoramento, tal tendência não deveria ser constatada. A maioria das
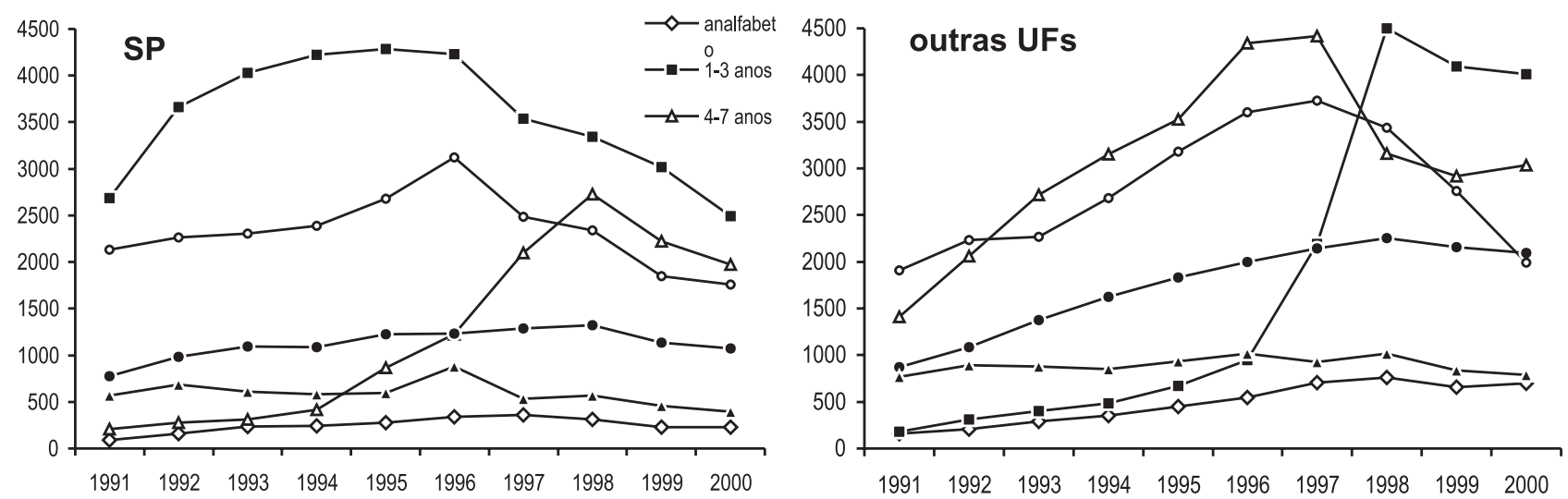

Figura 2 - Incidência de AIDS segundo anos de estudo. Estado de São Paulo e demais Unidades Federadas. Brasil. 1991-2000.
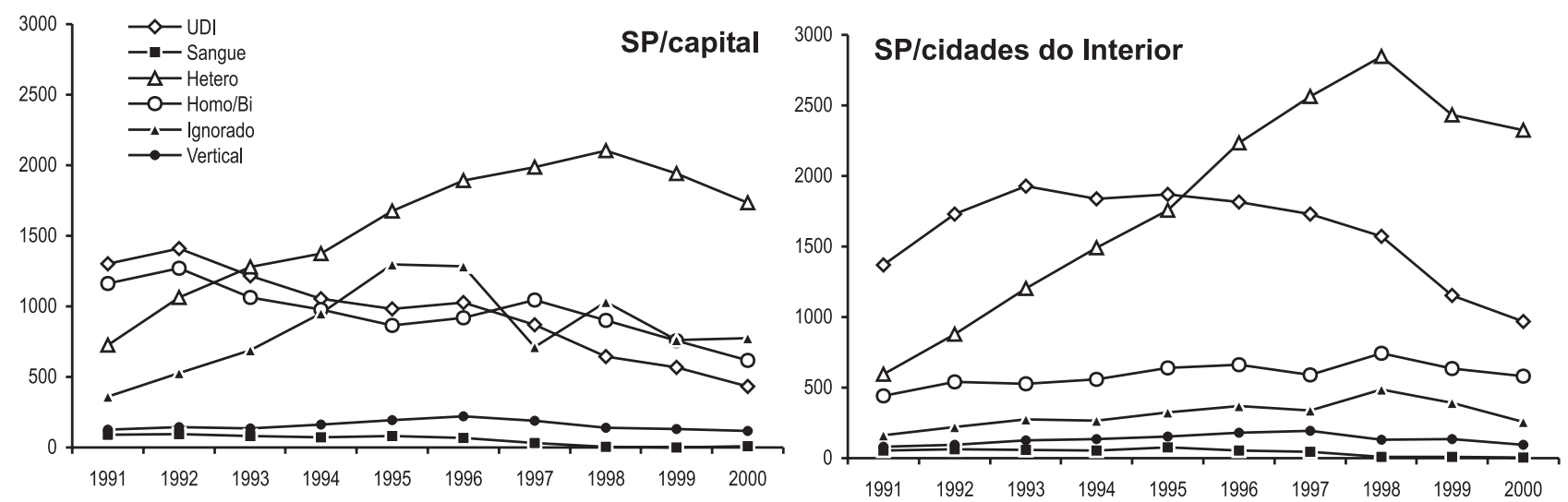

Figura 3 - Incidência de AIDS por categoria de transmissão e pelo ano de diagnóstico. Capital e interior do Estado de São Paulo. 1991-2000. 

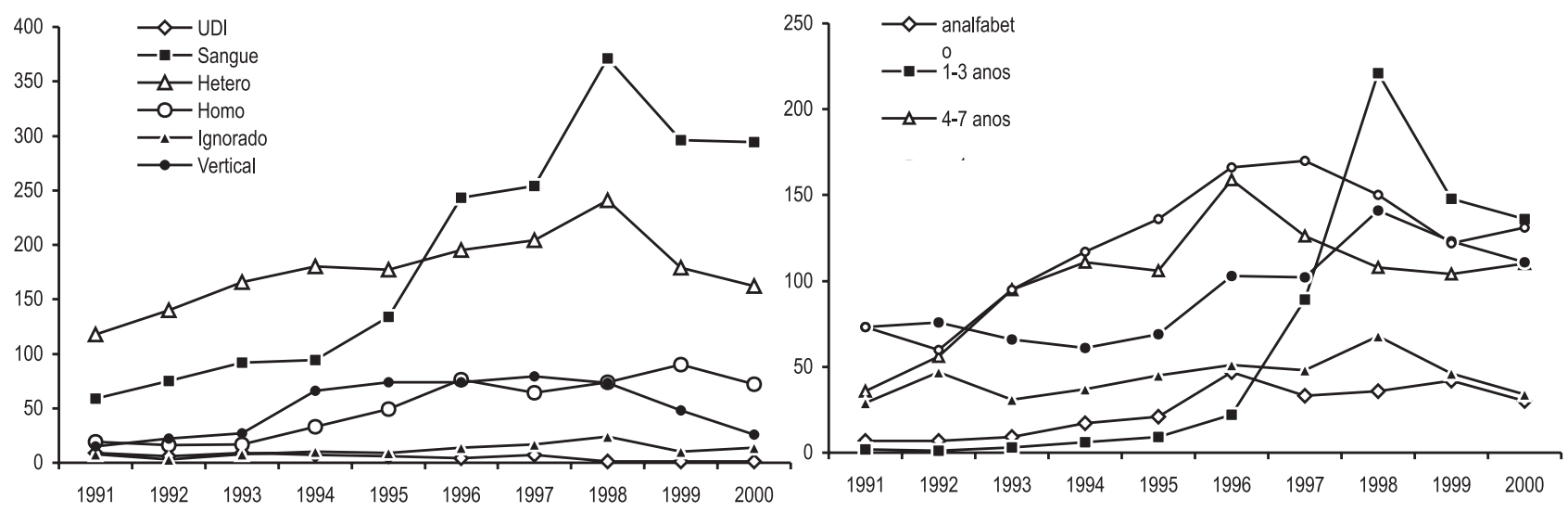

Figura 4 - Incidência de AIDS segundo ano de diagnóstico, categoria de transmissão e anos de estudo. Estado de Pernambuco. 1991-2000.
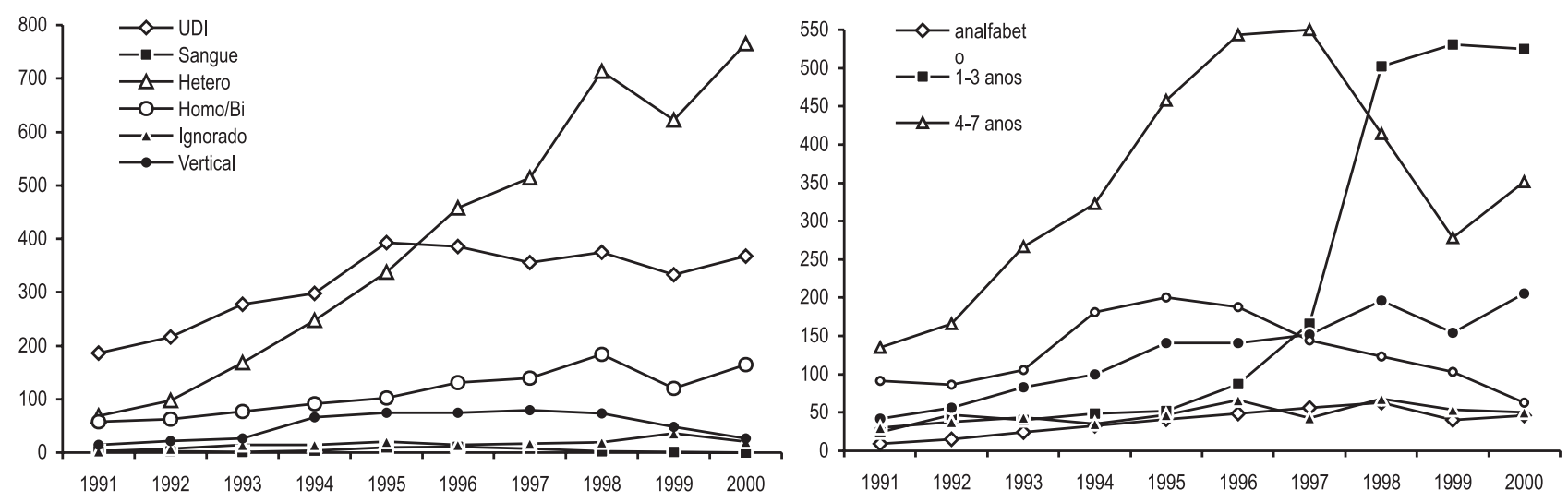

Figura 5 - Incidência de AIDS, por ano de diagnóstico, por categoria de transmissão e anos de estudo. Estado de Santa Catarina. 1991-2000.

notificações nesta categoria foi do sexo masculino com baixo grau de escolarização, além da parcela expressiva com escolaridade ignorada, constatando que 0 sistema de vigilância epidemiológica apresenta, além da subnotificação e do atraso de notificação, 0 viés de classificação.

Aconstatação de que o número de casos aumentou nos estratos de menor escolaridade remetem à condição de pior cobertura dos sistemas de vigilância e de assistência médica, entre os menos favorecidos economicamente, sob a hipótese de que a escolaridade é uma variável proxis importante de estratificação social.

Proporções maiores de casos de UDI nas regiões Centro-Oeste, Sudeste e Sul corroboram a tese de que a oferta e 0 acesso às drogas ilícitas são frequientes nestas regiões ${ }^{315}$. Contribuem para este fato a rota de escoamento do tráico, em direção aos portos e aeroportos, que, pelo barateamento da droga para facilitar a distribuição, atingem indivíduos de baixa condição socioeconômica ${ }^{11}$. Os Estados de São Paulo e de Santa Catarina participam deste cenário. 0 uso de drogas injetáveis com 0 compartilhamento de agulhas/seringas é um fator de risco importante de transmissão de AIDS. 0 aumento do número de casos notificados com baixa escolarização ratifica 0 fenômeno de pauperização ${ }^{7}$. 0 perfil encontrado para 0 Estado de Pernambuco, até então, mostrando-se similar àquele traço nacional do início da epidemia, sugere a necessidade de estudos visando esclarecer o por quê nem todas as áreas brasileiras não apresentam uma nítida heterossexualização da epidemia.

\section{AGRADECIMENTOS}

À Coordenação Nacional de DST e AIDS do Ministério da Saúde, pelo fornecimento do banco de dados.

\section{REFERÊNCIAS BIBLIOGRÁFICAS}

1. Bastos FI, Barcellos C. Geografia social da aids no Brasil. Revista de Saúde Pública 29: 52-62, 1995.

2. Brito AM, Castilho EA, Szwarcwald CL. AIDS e infecção pelo HIV no Brasil: uma epidemia multifacetada. Revista da Sociedade Brasileira de Medicina Tropical 34: 207-217, 2000.

3. Castilho EA, Chequer P. A epidemia da aids no Brasil. In: A epidemia da aids no Brasil: situação e tendências. Coordenação Nacional de DST e Aids, Brasília, Ministério da Saúde, p.9-12, 1997.

4. Castilho EA, Chequer P, Szwarcwald CL. A aids no Brasil. In: Rouquayrol E, Almeida N (eds) Epidemiologia \& Saúde. Editora Médica e Científica, Rio de Janeiro, p.271-284, 1999.

5. Coordenação Nacional de DST e Aids.Boletim Epidemiológico. Ministério da Saúde, Brasil, XIII, 2000.

6. Esteve J, Benhamou E, Raymond L. Descriptive epidemiology. International Agency for Research on Cancer Scientific Publications, Lyon, 1994.

7. Fonseca MG, Bastos Fl, Derrico M, Andrade CLT, Travassos C, Szwarcwald CL. AIDS e grau de escolaridade no Brasil: evolução temporal de 1986 a 1996. Cadernos de Saúde Pública 16: 77-87, 2000. 
8. Mann J, Tarantola DJM, Netter TW. A aids no mundo. ABIA da Universidade Estadual do Rio de Janeiro, Editora Relime Dumará, Rio de Janeiro, 1993.

9. Parker R. Na contramão da aids: sexualidade, intervenção, política. ABIA, Rio de Janeiro, Editora 34, São Paulo, 2000.

10. Parker R, Camargo Jr KR. Pobreza e HIV/aids: aspectos antropológicos e sociológicos. Cadernos de Saúde Pública 16: 89-102, 2000.

11. Programa das Nações Unidas para o Desenvolvimento. Atlas do desenvolvimento humano no Brasil. [programa de computador]. IPEA/ FIBGE/FJP, Brasília, 1998.

12. Rodrigues EHG, Abath FGC. Doenças sexualmente transmissíveis em pacientes infectados com HIV/AIDS no Estado de Pernambuco,
Brasil. Revista da Sociedade Brasileira de Medicina Tropical 33: 47-52, 2000

13. Rothman KJ, Greenland S. Modern Epidemiology. Lippincott-Raven Publishers, Philadelphia, $2^{\text {nd }}$ edition, 1998.

14. Rouquayrol MZ, Almeida Filho N. Epidemiologia e Saúde. MEDSI Editora Médica e Científica Ltda, Rio de Janeiro, 5a edição, 1999.

15. Szwarcwald CL, Bastos FI, Andrade CLT, Castilho EA. Aids: 0 mapa ecológico do Brasil, 1982-1994. In: A epidemia da aids no Brasil: situação e tendências. Coordenação Nacional de DST e Aids, Ministério da Saúde, Brasília, p. 27-44, 1997. 